In Cres. Vol. $3 N^{\circ}$ 1: pp. 99-108, 2012

\title{
NIVEL DE CONOCIMIENTO Y APLICACIÓN DE PRECAUCIONES DE AISLAMIENTO HOSPITALARIO POR LA ENFERMERA*
}

\author{
LEVEL OF KNOWLEDGE AND APPLYING ISOLATION \\ PRECAUTIONS IN HOSPITALS BY THE NURSE
}

\begin{abstract}
Nilda Margot Marcelo Álvarez", Nélida Cantuarias Noriega ${ }^{2}$
\end{abstract}

\section{RESUMEN}

El presente estudio de tipo cuantitativo, descriptivo, correlacional de corte transversal, se realizó con el objetivo de determinar la relación entre el nivel de conocimiento sobre manejo de aislamiento hospitalario y la aplicación de precauciones de aislamiento hospitalario por la (el) Enfermera(o). El universo muestral fue de 63 enfermeras (os) que laboran en los servicios de emergencia y hospitalización. Se aplicaron dos instrumentos: Test Nivel de Conocimiento sobre manejo de aislamiento hospitalario y lista de cotejo para la aplicación de precauciones de aislamiento hospitalario. Los resultados obtenidos demuestran que el $73 \%$ del personal posee regular conocimiento, seguido del $14,3 \%$, que tiene buen conocimiento. El 38,1\% corresponde al personal que a veces aplica estas precauciones, seguido de lo que no aplican, en un $36,5 \%$; finalmente se ubica un $25,4 \%$ que aplica las precauciones de aislamiento hospitalario. Se encontró relación estadísticamente significativa entre las variables de estudio al aplicar el Chi cuadrado para independencia de factores con el $95 \%$ de significación.

PALABRAS CLAVE: Nivel de conocimiento, Aplicación de precauciones de aislamiento hospitalario.

* Recibido: 20 de enero del 2012; aceptado: 14 de junio del 2012.

1 Maestra en Enfermería, Mención: Salud del Adulto. Docente de la Escuela de Enfermería. Universidad Católica Los Ángeles de Chimbote. Publicó el Artículo "Pobladores reciben capacitación en presupuesto participativo" (01 Setiembre 2011), en el Diario la Industria de Chimbote.

2 Doctora en Enfermería. Coordinadora de la Maestría en Enfermería y Salud Pública. Universidad Católica Los Ángeles de Chimbote. 


\begin{abstract}
This descriptive, comparative, and cross-sectional study aimed to determine the relationship between the level of knowledge on handling of hospitable isolation and the implementation of hospital isolation precautions by the Nurse. The sample population consisted of 63 nurses who work the emergencies and hospitalization area. Two instruments were applied: The test of knowledge on hospital isolation handling and the check list for application of hospital isolation handling. The obtained results demonstrate that $73 \%$ of the personnel have average knowledge, followed by $14,3 \%$ that have good knowledge. $38,1 \%$ corresponds to staff that sometimes applies these precautions, followed by the ones who do not apply,36,5\%. Finally, 25,4\% applies isolation hospital precautions. It was found high relationship between the variables of study when applying Chi Square for independence of factors with $95 \%$ of significance level.
\end{abstract}

KEY WORDS: Level of knowledge, Application of hospital isolation precautions.

\title{
I. INTRODUCCIÓN
}

Los adelantos tecnológicos, la globalización y la industrialización a gran escala han impuesto nuevos peligros y riesgo al personal que labora en las organizaciones. En las instituciones de salud estos riesgos se han incrementado considerablemente, es por ello que el Sistema de Gestión de las Organizaciones de Salud, incluye los riesgos hospitalarios y de los servicios de salud, con el propósito de controlarlos y proteger así al personal y a los pacientes. ${ }^{1}$

El ambiente animado del hospital consiste en los usuarios y quienes lo cuidan. El personal, los usuarios, los visitantes y la comunidad en general constituyen el principal reservorio infeccioso del hospital, contaminando el ambiente con los microorganismos de la flora normal o patógena de la piel y de las mucosas que forman la cavidad bucal, faríngea y nasal, o con las bacterias de sus secreciones o excreciones. ${ }^{2}$

Es imposible identificar a todas las personas con algún microorganismo infeccioso, por lo que se recomienda considerar a todos los usuarios como potencialmente infecciosos. Además, el riesgo de infección va a ser proporcional a la prevalencia de la enfermedad de la población asistida y a la probabilidad de producción de accidentes durante la realización de los procedimientos. ${ }^{3}$

Frente a esta realidad, las precauciones de aislamiento hospitalario deben ser una práctica rutinaria en los servicios y ser cumplidas por todo el personal que labora en éstos, independientemente del grado de riesgo según su actividad y las dife- 
rentes áreas que componen los establecimientos de salud. Tienen como propósito básico que el personal de salud no se contagie ni contagie; por lo tanto, debe entenderse como una doctrina encaminada a disminuir el riesgo al paciente y al trabajador de salud de adquirir enfermedades intrahospitalarias. ${ }^{4,5}$

El profesional de enfermería cumple una importante función en la reducción de riesgos mediante la aplicación de las precauciones de aislamiento hospitalario. El riesgo de infección se incrementa en forma considerable a medida que el equipo técnico asociado con la atención del paciente se hace más complejo y a medida que se utilizan más dispositivos que interrumpen las barreras de protección anatómicas naturales. $^{6}$

Sin embargo, el trabajo diario del profesional de Enfermería en las instituciones de Salud se realiza con exceso de confianza y sólo se cuidan de usuarios diagnosticados con enfermedades contagiosas; o cuando vemos que los desechos del establecimiento se recogen y eliminan sin medidas de bioseguridad, afrontando ante situaciones cotidianas que entrañan un grave peligro a su salud y a la salud de quienes cuidan. ${ }^{2}$

El nivel de conocimientos es el grado de percepción o entendimiento referente a cierto tema o fenómeno adquirido a través del aprendizaje o la investigación. Conocer las medidas de aislamiento hospitalario es una responsabilidad y de conducta del profesional de enfermería que hace posible aplicarlas en forma correcta en la práctica, brindando cuidados de enfermería en forma científica y holística de salud. ${ }^{7}$

Dávila, B. y otros, el 2001, ${ }^{8}$ en su estudio realizado concluyen que los médicos muestran un nivel de conocimiento insuficiente; en relación con los indicadores de salud, el 3\% considera que las infecciones intrahospitalarias tienen poca relación con las prácticas del personal que atiende a los usuarios.

En Juliaca, departamento de Puno, en un estudio comparativo entre el nivel de conocimiento y actitudes de los médicos y paramédicos del Hospital Carlos Monje Medrano (MINSA) y el Hospital del IPSS de Juliaca, en 1997, se encontró buen nivel de conocimiento $82 \%$ y $87 \%$, respectivamente, en ambos lugares y en los distintos grupos ocupacionales, por lo que concluye que los conocimientos están relacionados con las actitudes en ambos hospitales. ${ }^{9}$

Es necesario aplicar las precauciones de aislamiento hospitalario en el cuidado del usuario hospitalizado con o sin diagnóstico de infección, considerando una de las principales medidas de prevención para disminuir el riesgo de contraer enfermedades infecto contagiosas y de cortar la cadena de transmisión del patógeno, al estar en contacto con el ambiente, vías aéreas, fluidos corpora- 
les, equipos y dispositivos utilizados en los cuidados de enfermería, procedimientos de tratamientos invasivos y de diagnóstico. ${ }^{10}$

Por ello, para involucrar al profesional de Enfermería en la aplicación de las precauciones de aislamiento hospitalario se necesita que tenga los conocimientos básicos de aislamiento hospitalario y normas de prevención y control de infecciones intrahospitalarias, motivo del presente trabajo de investigación. ${ }^{10}$

\section{PROBLEMA}

¿Cuál es la relación entre el nivel de conocimientos y la aplicación de precauciones de aislamiento hospitalario por la (el) Enfermera(o) en el Hospital Regional Eleazar Guzmán Barrón - Nuevo Chimbote, 2007 ?

\section{OBJETIVOS}

El presente estudio tiene como objetivo general determinar la relación entre el nivel de conocimientos sobre manejo de aislamiento hospitalario y la aplicación de precauciones de aislamiento hospitalario por la (el) enfermera(o) del Hospital Regional Eleazar Guzmán Barrón - Nuevo Chimbote.

Los objetivos específicos son:

a) Identificar la aplicación de precauciones de aislamiento hospitalario por la (el) enfermera(o) del Hospital Regional Eleazar Guzmán Barrón.

b) Determinar el nivel de conocimientos sobre manejo de aislamiento hospitalario en el (la) enfermero(a) del Hospital Regional Eleazar Guzmán Barrón.

El marco teórico se ha desarrollado teniendo en cuenta los enfoques de la teoría social del aprendizaje de Albert Bandura, así como las bases conceptuales del aislamiento hospitalario.

La Teoría del Aprendizaje Social, de Albert Bandura, se centra en los conceptos de refuerzo y observación que sostienen que los humanos adquieren destrezas y conductas de modo operante e instrumental y que entre la observación y la imitación intervienen factores cognitivos que ayudan al sujeto a decidir si lo observado se imita o no. ${ }^{11}$

Los factores cognitivos se refieren concretamente a la capacidad de reflexión y simbolización, así como a la prevención de consecuencias basadas en procesos de comparación, generalización y autoevaluación. En definitiva, el 
comportamiento depende del ambiente, así como de los factores personales (motivación, retención y producción motora) y cognitivos (conocimiento del tema). ${ }^{11}$

El aprendizaje observacional, según Bandura, ${ }^{17}$ demuestra que los seres humanos adquieren conductas nuevas sin un reforzado obvio y hasta cuando carecen de la oportunidad para aplicar el conocimiento. El único requisito para el aprendizaje puede ser que la persona observe a otro individuo o modelo, llevar a cabo una determinada conducta. El comportamiento no se desarrolla exclusivamente a través de lo que aprende el individuo directamente por medio del acondicionamiento operante y clásico, sino también a través de lo que aprende indirectamente (vicariamente) mediante la observación y la representación simbólica de otras personas y situaciones. ${ }^{11,12}$

El sistema de aislamiento son procedimientos destinados a cortar la cadena de transmisión de patógenos productores de las infecciones intrahospitalarias y se basa en dos tipos de precauciones: Precauciones estándar y precauciones basadas en el mecanismo de transmisión. ${ }^{13}$

Las precauciones estándar son los procedimientos que buscan disminuir el riesgo de transmisión de microorganismos de cualquier fuente hospitalaria y resumen las precauciones universales y el aislamiento de sustancias corporales. Se aplican en todas las situaciones en las que se manipule sangre, fluidos corporales, secreciones y excreciones (excepto sudor, piel no intacta y mucosas) y los elementos punzantes y cortantes en el manejo de todos los pacientes hospitalizados con o sin diagnóstico de infección. ${ }^{14}$

La aplicación de las medidas de precauciones de aislamiento hospitalario es uno de los aspectos controversiales en salud; es el manejo de pacientes infectados en los hospitales. Para este fin se ha diseñado el sistema de aislamiento hospitalario, siendo el instrumento que el profesional de enfermería deberá de utilizar de manera rutinaria, aplicando estas precauciones que se basan en medidas simples de fácil aprendizaje y manejo en el cuidado del paciente para prevenir riesgos de transmisión de infecciones entre un paciente y otro y entre el paciente y el profesional de enfermería y viceversa, durante su recuperación y reincorporación a su entorno familiar y social. ${ }^{15}$

\section{METODOLOGÍA}

El presente estudio de investigación es de tipo cuantitativo, descriptivo, correlacional, de corte transversal. ${ }^{16}$ 
La población muestral es el profesional de Enfermería que labora en los servicios de Emergencia y Hospitalización del Hospital Regional Eleazar Guzmán Barrón, de Nuevo Chimbote, constituida por un total de 63 enfermeras (os). La unidad de análisis, la (el) enfermera(o) que labora en el Hospital Regional Eleazar Guzmán Barrón y que cumplan con los criterios de inclusión.

La variable independiente: Nivel de conocimientos sobre manejo de aislamiento hospitalario, está definida como el grado de perfección o entendimiento referente a cierto tema o fenómeno, adquirido a través del aprendizaje o la investigación.

La variable dependiente: Aplicación de precauciones de aislamiento hospitalario, definida como el conjunto de precauciones de aislamiento hospitalario aplicadas para el cuidado del usuario y del profesional de Enfermería, destinados a cortar la cadena de transmisión de microorganismos productores de infecciones intrahospitalarias. ${ }^{2}$

Los datos del cuestionario y de la lista de cotejo fueron procesados con el programa SPSS V. 14, y los resultados fueron presentados en cuadros de doble entrada a nivel de frecuencia absoluta y relativa porcentual. Se empleó algunos gráficos de barras para variables consideradas relevantes al estudio.

Para determinar si existe relación o asociación entre las variables de estudio se aplicó la prueba chi cuadrado para independencia de factores. Si P $<0,05$, las variables o factores están relacionados. De existir asociación, su nivel fue medido empleando el coeficiente de contingencia (C), que toma valores entre 0 y 1 . Cuando más próximo se encuentre a uno, la relación será muy fuerte.

\section{RESULTADOS Y DISCUSIÓN}

Tabla 01

NIVEL DE CONOCIMIENTOS SOBRE MANEJO DE AISLAMIENTO HOSPITALARIO. HOSPITAL REGIONAL ELEAZAR GUZMÁN BARRÓN. NUEVO CHIMBOTE, 2007

\begin{tabular}{lcc}
\hline Nivel de Conocimientos & $\mathbf{N}^{\circ}$ & $\%$ \\
\hline Bueno & 9 & 14,3 \\
Regular & 46 & 73,0 \\
Deficiente & 8 & 12,7 \\
\hline TOTAL & 63 & 100,0 \\
\hline
\end{tabular}

FUENTE: Test cuestionario de nivel de conocimientos sobre manejo de aislamiento hospitalario. Marcelo, N. Nuevo Chimbote, mayo 2007. 
En los resultados de la Tabla 01 podemos observar que el $73 \%$ del personal posee regular conocimiento sobre el manejo de aislamiento hospitalario, seguido de los que poseen buen conocimiento, quienes logran el 14,3\% y con deficiente nivel de conocimiento, un $12,7 \%$.

Tabla 02

APLICACIÓN DE PRECAUCIONES DE AISLAMIENTO

HOSPITALARIO. HOSPITAL REGIONAL ELEAZAR

GUZMÁN BARRÓN. NUEVO CHIMBOTE, 2007

\begin{tabular}{lcc}
\hline $\begin{array}{l}\text { Aplicaciones de Precauciones } \\
\text { de Aislamiento }\end{array}$ & $\mathbf{N}^{\circ}$ & $\%$ \\
\hline Si aplica & 16 & 25,4 \\
A veces aplica & 24 & 38,1 \\
No aplica & 23 & 36,5 \\
\hline TOTAL & 63 & 100,0 \\
\hline
\end{tabular}

FUENTE: Lista de cotejo para la aplicación de precauciones de aislamiento hospitalario. Marcelo, N. Nuevo Chimbote, mayo 2007.

Según los resultados de la tabla 02 , el mayor porcentaje $(38,1 \%)$ corresponde al personal que a veces aplica las precauciones de aislamiento hospitalario, seguido de los que no aplican (36,5\%); finalmente se ubica un $25,4 \%$ que aplica.

Tabla 03

RELACIÓN ENTRE EL NIVEL DE CONOCIMIENTOS Y LA APLICACIÓN DE PRECAUCIONES DE AISLAMIENTO HOSPITALARIO

\begin{tabular}{|c|c|c|c|c|c|c|c|c|}
\hline \multirow{3}{*}{$\begin{array}{l}\text { Nivel de } \\
\text { Conocimientos }\end{array}$} & \multicolumn{6}{|c|}{ Aplicación de Precauciones de Aislamiento Hospitalario } & \multirow{2}{*}{\multicolumn{2}{|c|}{ Total }} \\
\hline & \multicolumn{2}{|c|}{ Aplica } & \multicolumn{2}{|c|}{ A Veces Aplica } & \multicolumn{2}{|c|}{ No Aplica } & & \\
\hline & $\mathbf{N}^{\circ}$ & $\%$ & $\mathbf{N}^{\circ}$ & $\%$ & $\mathbf{N}^{\circ}$ & $\%$ & $\mathbf{N}^{\circ}$ & $\%$ \\
\hline Bueno & 6 & 9,5 & 0 & 0 & 3 & 4,8 & 9 & 14,3 \\
\hline Regular & 10 & 15,9 & 24 & 38,1 & 12 & 19,0 & 46 & 73,0 \\
\hline Deficiente & 0 & 0 & 0 & 0 & 8 & 12,7 & 8 & 12,7 \\
\hline TOTAL & 16 & 25,4 & 24 & 38,1 & 23 & 36,5 & 63 & 100 \\
\hline
\end{tabular}

Fuente: Test cuestionario de nivel de conocimientos sobre manejo de aislamiento hospitalario. Marcelo, N. Nuevo Chimbote, mayo 2007.

$\mathrm{x}^{2}=24,99 \quad \mathrm{gl}=4 \quad \mathrm{P}=0,000001 \quad \mathrm{C}=53,3 \%$ 
$\mathrm{Al}$ aplicar la prueba de Chi cuadrado para independencia de factores, se obtuvo un valor P menor de 0,001 , lo cual indica existencia de alta relación entre el nivel de conocimiento y la aplicación. Esta relación es del 53,3\%, lo que confirma la hipótesis.

Los resultados del presente estudio denotan lo esencial que son los conocimientos para la práctica cotidiana de estas normas y medidas de precaución, la cual debe ser integrada al quehacer diario del profesional de enfermería, adquiriendo los conocimientos para aplicarlos a través de la retroalimentación y/o la capacitación del personal nuevo en su proceso de inducción al cuidado asistencial en el hospital. ${ }^{17}$

Como se observa, el nivel de conocimiento de la mayoría de enfermeras es regular, con el $73 \%$, probablemente debido a factores que condicionan la aplicación de medidas de aislamiento hospitalario, como la falta de información, la capacitación o un programa de actualización y la actitud del profesional de enfermería ante los riesgos de infecciones intrahospitalarias, lo que demuestra la necesidad de desarrollar una sólida base de conocimientos que moldean las competencias intelectuales y las habilidades de cada enfermera.

Los resultados de los estudios de investigación muestran que la aplicación de las precauciones del aislamiento hospitalario son medidas de prevención destinadas a cortar la cadena de transmisión de patógenos productores de infecciones intrahospitalarias. Esta debe ser una práctica diaria y ser cumplida por el profesional de enfermería que tiene a su cargo los cuidados del paciente, independiente del grado de riesgo en los diferentes servicios del hospital. ${ }^{13}$

Los conocimientos y la aplicación de las precauciones de aislamiento hospitalario buscan disminuir el riesgo de transmisión de microorganismos de cualquier fuente hospitalaria y se aplican en el manejo de todos los pacientes con o sin diagnóstico documentado o se sospecha de una infección de importancia epidemiológica, indispensable en la prevención y control de infecciones intrahospitalarias, que viene siendo un problema actual y en constante evolución, cuyo denominador común es haber sido adquirido en un hospital. ${ }^{18,19}$

El nivel de conocimiento sobre precauciones de aislamiento hospitalario y su aplicación significa cumplir con responsabilidad las normas y principios que deben regir el actuar del profesional de enfermería durante su ejercicio profesional al brindar cuidados de salud. ${ }^{13}$

Al contrastar los resultados observamos que un profesional de enfermería debe ser consciente del problema de las infecciones y sus efectos en la morbimortalidad, por lo que es indispensable tener conocimiento sobre los tipos de 
infecciones que se presentan con mayor frecuencia, los agentes patógenos comunes y cómo se trasmiten; es decir, una enfermera(o), al estar capacitada sabe cuál es la forma de protegerse a sí mismo y a sus pacientes, mostrando una actitud positiva en los cuidados, siendo los mejores medios para la práctica de medidas de precauciones de aislamiento hospitalario en el cuidado integral del paciente necesarios para disminuir las infecciones intrahospitalarias. ${ }^{19}$

\section{CONCLUSIONES}

1. La tercera parte de las (los) enfermeras(os) a veces aplica las precauciones de aislamiento hospitalario, seguido de un $36,5 \%$, que no aplica, y un $25 \%$, que aplica.

2. La mayoría de las (los) enfermeras(os) posee regular nivel de conocimientos sobre manejo de aislamiento hospitalario, seguido de un $14,3 \%$, que tiene buen conocimiento.

3. Existe alta relación estadísticamente significativa entre el nivel de conocimiento y la aplicación de aislamiento hospitalario, lo cual se estableció al aplicar la prueba de Chi cuadrado para la independencia de factores, obteniendo un valor $\mathrm{p}<0,001$.

\section{REFERENCIAS BIBLIOGRÁFICAS}

1 Henderson V. Nite N. Enfermería teórica y práctica. En: Cuidados básicos de enfermería en la atención del paciente. 6ta. edición. Editorial La Prensa Médica Mexicana S.A. México. 1998.

2 Ministerio De SAlud. Bioseguridad. Programa Salud Básica para todos. Ministerio de Salud. Perú. 1997.

3 Ministerio de SAlud. Oficina General de Epidemiología. Proyecto Vigía. Protocolo para el estudio de conocimientos, actitudes y prácticas del personal de salud en el control de infecciones intrahospitalarias. Perú. 2002.

4 EsSalud. Normas Generales de Bioseguridad en EsSalud. Centro de Prevención de riesgos de trabajo. Perú. 2001.

5 GARCÍA, A. Medicina de Bioseguridad e Infección Intrahospitalaria. Revista de enfermería IMSS, 2002, 10 (1) 27-30 URL: http://www.gob.Mx/NR/rdonlyres/2E34C3FF-A527-42F1-B7219EE6DFF24C47/0/medidas20.pdf.

6 Smeltzer, BARE B. Enfermería Médico Quirúrgica. 9na. edición. Editorial Mc Graw-Hill Interamericana Editores S.A. de C. V. México. V. II. 2002.

7 Contreras, A. Teoría del aprendizaje Social Cognitiva - Albert Bandura, 2001. URL: http://www.comminit.com/la/modelosdeplaneacion/lapm/lasld-578.html.

8 Cachihuaman F., DÁvila J., Barriga A. Y Otros. Conocimientos y Actitudes de los Médicos frente a las Infecciones Intrahospitalarias - Universidad Peruana Cayetano Heredia. 2001. URL: http://143.107,183.180/recuadros/ME108. 
9 CONDORI J. P. Estudio Comparativo de Conocimientos y Actitudes relacionadas al VIH / SIDA y sus Normas de Bioseguridad entre el personal médico y paramédico del Hospital Carlos Monge Medrano y el Hospital del IPSS Juliaca. (Tesis de Bachiller Medicina). Arequipa: Universidad Nacional San Agustín. Perú. 1997.

10 SoRnMA, Incertidumbre y Riesgos en Bioseguridad y sus Implicaciones para las Políticas Ambientales en América Latina. 2002. Noviembre. URL: http://www.infecto.edu.uy/prevencion/ bioseguridad/bioseguridad.htm.

11 Bandura, A. Temas de Estudios, 2006. URL: http://www.temas-estudios.com/albert_ bandura teoría.aspBoeree.

12 Broto M., Delor S., SAlinas J. Instrumentación Quirúrgica - Técnicas en Cirugía General. Editorial Panamericana S.A. España. V. VI. 2000.

13 Ministerio de SALud. Actualizaciones en Aislamiento. Circular 46. Perú. 2000.

14 Soto C., Olano D. Conocimiento de las Normas de Bioseguridad por el personal asistencial del Hospital Nacional Almanzor Aguinaga Asenjo. Trabajo de Investigación Cátedra de Medicina Preventiva. Lambayeque: Facultad de Medicina Humana, Universidad Nacional Pedro Ruiz Gallo. Perú.2001.

15 Ministerio de SALud - USAID - VIGÍA. Curso de Prevención y Control de las Infecciones Intrahospitalarias para profesionales. Organizado por la Dirección General de Salud para todas las personas y la Dirección de Salud - Lambayeque, Chiclayo - Perú. 2003.

16 Cuyubamba, N. Estudio de Relación entre los Niveles de Conocimiento y Actitudes del personal de Salud, para la Aplicación de las Medidas de Bioseguridad en los Servicios de Mayor Riesgo del Hospital Félix Mallorca Soto. Perú. 2003. Ministerio de Salud. Actualizaciones en Aislamiento. Circular 46. Perú. 2000.

17 MAlagón, Londoño, HeRnÁNDEZ E. Infecciones Hospitalarias. 2da. edición. Editorial Médica Internacional Ltda. Colombia. 1999.

18 Ministerio de SALUd. Prevención de Infecciones por Virus Respiratorios en Pediatría. Circular 4 C/18. Perú. 1999.

19 Ministerio de SAlud - USAID - ViGíA. Curso de Prevención y Control de las Infecciones Intrahospitalarias para profesionales. Organizado por la Dirección General de Salud para todas las personas y la Dirección de Salud - Lambayeque, Chiclayo - Perú. 2003. 